\title{
A NOTE ON THE PROBABILITY OF GENERATING ALTERNATING OR SYMMETRIC GROUPS
}

\author{
LUKE MORGAN AND COLVA M. RONEY-DOUGAL
}

\begin{abstract}
We improve on recent estimates for the probability of generating the alternating and symmetric groups $\mathrm{A}_{n}$ and $\mathrm{S}_{n}$. In particular we find the sharp lower bound, if the probability is given by a quadratic in $n^{-1}$. This leads to improved bounds on the largest number $h\left(\mathrm{~A}_{n}\right)$ such that a direct product of $h\left(\mathrm{~A}_{n}\right)$ copies of $\mathrm{A}_{n}$ can be generated by two elements.
\end{abstract}

\section{INTRODUCTION}

For a group $X=\mathrm{S}_{n}$ or $\mathrm{A}_{n}$, we write $p(X)$ for the probability that two elements of $X$ generate a group that contains $\mathrm{A}_{n}$. In [1], Dixon proved that $p\left(\mathrm{~S}_{n}\right) \rightarrow 1$ as $n \rightarrow \infty$. In [2] he sharpened this statement to

$$
p\left(\mathrm{~S}_{n}\right)=1-\frac{1}{n}-\frac{1}{n^{2}}-\frac{4}{n^{3}}-\frac{23}{n^{4}}-\frac{171}{n^{5}}-\frac{1542}{n^{6}}+O\left(n^{-7}\right) .
$$

For many applications, numerical results are needed, rather than asymptotics. In [5] Maróti and Tamburini proved explicit upper and lower bounds

$$
1-\frac{1}{n}-\frac{13}{n^{2}}<p(X) \leqslant 1-\frac{1}{n}+\frac{2}{3 n^{2}} .
$$

In this present note, we find the best possible lower bound of this type, and a close-to-optimal upper bound.

Theorem 1.1. Let $X=\mathrm{A}_{n}$ or $X=\mathrm{S}_{n}$ with $n \geqslant 5$. Then

$$
1-\frac{1}{n}-\frac{8.8}{n^{2}} \leqslant p(X)<1-\frac{1}{n}-\frac{0.93}{n^{2}} .
$$

Equality holds in the lower bound if and only if $n=6$.

In fact, for $n \geqslant 14$, we prove that $1-\frac{1}{n}-\frac{7.5}{n^{2}}<p(X)<1-\frac{1}{n}-\frac{0.93}{n^{2}}$. The result for smaller $n$ comes from the values for $p(X)$ in Table 1(taken from [7, Table 4.1]).

Hall [3] considered the largest number $h(S)$ such that a direct product of $h(S)$ copies of a non-abelian finite simple group $S$ can be generated by two elements, and proved that $h(S)=p(S)|S| / \mid$ Out $(S) \mid$. The function $h(S)$ has received considerable attention recently; we refer the reader to [5] for more discussion and references and to [6] for lower bounds on $h(S)$ for all non-abelian finite simple groups $S$. The new bounds above yield:

Corollary 1.2. Let $n$ be an integer with $n \geqslant 14$. Then

$$
\left(1-\frac{1}{n}-\frac{7.5}{n^{2}}\right)\left(\frac{n !}{4}\right)<h\left(\mathrm{~A}_{n}\right)<\left(1-\frac{1}{n}-\frac{0.93}{n^{2}}\right)\left(\frac{n !}{4}\right) .
$$

Let $m(S)$ denote the minimal index of a proper subgroup of a group $S$. In [4, it is proved that there exist absolute constants $c_{1}$ and $c_{2}$ such that $1-c_{1} / m(S)<p(S)<1-c_{2} / m(S)$, for all non-abelian finite simple groups $S$. Our main theorem immediately yields the following bounds on $c_{1}$ and $c_{2}$.

Corollary 1.3. For $n \geqslant 5$,

$$
1-\frac{2.468}{n}<p\left(\mathrm{~A}_{n}\right)<1-\frac{1.186}{n}
$$

and hence $c_{1} \geqslant 2.468$ and $c_{2} \leqslant 1.186$.

1991 Mathematics Subject Classification. 20B30; $20 \mathrm{P} 05$.

Key words and phrases. Symmetric group; alternating group; generation; probability.

The research of the first author is supported by the Australian Research Council grant DP120100446. This work was done whilst the second author was visiting The University of Western Australia as a Cheryl E. Praeger Visiting Research Fellow. The authors would like to thank the anonymous referee for their extremely helpful remarks. 


\section{Proof of Theorem 1.1}

Definition 2.1. For $X=\mathrm{A}_{n}$ or $\mathrm{S}_{n}$ we let $p_{\text {intrans }}(X)$ and $p_{\text {trans }}(X)$ be the probability that two elements chosen randomly from $X$ generate a subgroup of an intransitive maximal subgroup of $X$, or a subgroup of a transitive maximal subgroup of $X$ other than $\mathrm{A}_{n}$, respectively.

Lemma 2.2. Let $X=\mathrm{A}_{n}$ or $\mathrm{S}_{n}$ with $n \geqslant 14$. Then

$$
p_{\text {intrans }}(X)<\frac{1}{n}+\frac{2.7}{n^{2}} \text {. }
$$

Proof. We prove the result for $\mathrm{S}_{n}$, the arguments for $\mathrm{A}_{n}$ are identical. Let $x, y \in \mathrm{S}_{n}$ and suppose that $Y:=\langle x, y\rangle$ is contained in an intransitive maximal subgroup. Then $Y$ is contained in a subgroup conjugate to $\mathrm{S}_{k} \times \mathrm{S}_{n-k}$ for some $1 \leqslant k \leqslant\left\lfloor\frac{n-1}{2}\right\rfloor$.

Let $k \in\{1, \ldots, n-1\}$. Then the probability that $Y \leqslant \mathrm{~S}_{k} \times \mathrm{S}_{n-k}$ is bounded by

$$
\left(\begin{array}{l}
n \\
k
\end{array}\right)\left(\frac{k !(n-k) !}{n !}\right)^{2}=\left(\begin{array}{l}
n \\
k
\end{array}\right)^{-1}
$$

So the probability that $Y \leqslant \mathrm{~S}_{1} \times \mathrm{S}_{n-1}$ is at most $\frac{1}{n}$, and the probability that $Y \leqslant \mathrm{~S}_{2} \times \mathrm{S}_{n-2}$ and $Y$ is transitive on the orbit of size 2 is bounded by

$$
\frac{3}{4} \frac{2}{n(n-1)}=\frac{3}{2 n(n-1)} .
$$

Similarly, the probability that $Y \leqslant \mathrm{~S}_{3} \times \mathrm{S}_{n-3}$ and $Y$ is transitive on the orbit of length 3 is

$$
\frac{13}{18}\left(\begin{array}{c}
n \\
3
\end{array}\right)^{-1}=\frac{13}{3 n(n-1)(n-2)} .
$$

Now the probability that $Y \leqslant \mathrm{~S}_{k} \times \mathrm{S}_{n-k}$ for some $4 \leqslant k \leqslant\left\lfloor\frac{n-1}{2}\right\rfloor$ is

$$
\sum_{k=4}^{\left\lfloor\frac{n-1}{2}\right\rfloor} \frac{1}{\left(\begin{array}{l}
n \\
k
\end{array}\right)} \leqslant \sum_{k=4}^{\left\lfloor\frac{n-1}{2}\right\rfloor} \frac{1}{\left(\begin{array}{l}
n \\
4
\end{array}\right)} \leqslant \frac{12(n-7)}{n(n-1)(n-2)(n-3)} .
$$

We now observe that, since $n \geqslant 14$,

$$
\frac{3}{2 n(n-1)}+\frac{13}{3 n(n-1)(n-2)}+\frac{12(n-7)}{n(n-1)(n-2)(n-3)}<\frac{2.7}{n^{2}}
$$

which completes the proof.

Lemma 2.3. Let $X=\mathrm{A}_{n}$ or $\mathrm{S}_{n}$, with $n \geqslant 14$. Then

$$
p_{\text {intrans }}(X)>\frac{1}{n}+\frac{0.93}{n^{2}} \text {. }
$$

Proof. We observe that $p_{\text {intrans }}(X)$ is bounded below by the probability that a random pair of elements of $X$ generate a subgroup with a fixed point, or with an orbit of size 2 . For $X=\mathrm{S}_{n}$, we bound $p_{\text {intrans }}(X)$ by doing inclusion-exclusion to depth 2 on the union of the sets $\left(\mathrm{S}_{n}\right)_{\alpha}$, with $1 \leqslant \alpha \leqslant n$, and $\left(\mathrm{S}_{n}\right)_{\{\alpha, \beta\}} \backslash\left(\mathrm{S}_{n}\right)_{(\alpha, \beta)}$, with $1 \leqslant \alpha<\beta \leqslant n$. We find that $p_{\text {intrans }}(X)$ is greater than

$$
\frac{1}{n}+\frac{3}{4} \frac{2(n-2) !}{n !}-\frac{(n-2) !}{2 n !}-\frac{3}{4}\left(\begin{array}{c}
n \\
1
\end{array}\right)\left(\begin{array}{c}
n-1 \\
2
\end{array}\right)\left(\frac{2(n-3) !}{n !}\right)^{2}-\left(\frac{3}{4}\right)^{2} \frac{\left(\begin{array}{c}
n \\
2
\end{array}\right)\left(\begin{array}{c}
n-2 \\
2
\end{array}\right)}{2}\left(\frac{4(n-4) !}{n !}\right)^{2}
$$

Thus

$$
p_{\text {intrans }}(X) \geqslant \frac{1}{n}+\frac{8 n^{2}-52 n+75}{8 n(n-1)(n-2)(n-3)}
$$

which, since $n \geqslant 14$, is greater than $\frac{1}{n}+\frac{0.93}{n^{2}}$.

Proof of Theorem 1.1. For the upper bound we use Lemma 2.3. For the lower bound, note that

$$
1-p(X)=p_{\text {intrans }}(X)+p_{\text {trans }}(X) .
$$

It follows from the proofs of [5, Lemmas 3.1 and 4.3] that $p_{\text {trans }}(X) \leqslant \frac{4.8}{n^{2}}$. Combining this with Lemma 2.2 gives the theorem.

In Table 1 we record the value of $p\left(\mathrm{~A}_{n}\right)$ and $p\left(\mathrm{~S}_{n}\right)$ for $n \leqslant 13$, together with our lower and upper bounds as stated in Theorem 1.1. All values are correct to three decimal places. 
TABle 1. Precise values and bounds for $p(X)$

\begin{tabular}{c||c|c|c|c|c|c|c|c|c}
$n$ & 5 & 6 & 7 & 8 & 9 & 10 & 11 & 12 & 13 \\
\hline$p\left(\mathrm{~A}_{n}\right)=$ & 0.633 & 0.588 & 0.726 & 0.739 & 0.848 & 0.875 & 0.893 & 0.902 & 0.913 \\
$p\left(\mathrm{~S}_{n}\right)=$ & 0.633 & 0.588 & 0.795 & 0.796 & 0.859 & 0.875 & 0.894 & 0.903 & 0.913 \\
$p(X) \geqslant$ & 0.448 & 0.588 & 0.677 & 0.737 & 0.780 & 0.812 & 0.836 & 0.855 & 0.871 \\
$p(X) \leqslant$ & 0.763 & 0.808 & 0.839 & 0.861 & 0.878 & 0.891 & 0.902 & 0.911 & 0.918
\end{tabular}

\section{REFERENCES}

[1] J. D. Dixon. The probability of generating the symmetric group. Math. Z 110 (1969) 199-205.

[2] J. D. Dixon. Asymptotics of generating the symmetric and alternating groups. Electron. J. Combin. 12 (2005), Research paper 56, $5 \mathrm{pp}$.

[3] P. Hall. The Eulerian function of a group. Quart. J. Math. Oxford 7 (1936), 133-141.

[4] M. W. Liebeck \& A. Shalev. Simple groups, probabilistic methods, and a conjecture of Kantor and Lubotszky. J. Algebra 184 (1996) 31-57.

[5] A. Maróti \& M.C. Tamburini. Bounds for the probability of generating the symmetric and alternating groups. Arch. Math. (Basel) 96(2) (2011) 115-121.

[6] N. E. Menezes, M. Quick \& C. M. Roney-Dougal. The probability of generating a finite simple group. Israel J. Math 198 (2013) 371-392.

[7] N. E. Menezes. Random generation and chief length of finite groups. PhD thesis, University of St Andrews (2013).

Luke Morgan, School of Mathematics and Statistics,, University of Western Australia,, 35 Stirling Highway,, Crawley, WA 6009, Australia

E-mail address: luke.morgan@uwa.edu.au

Colva M. Roney-Dougal, School of Mathematics and Statistics,, University of St Andrews,, North Haugh,, St Andrews,, Fife KY16 9SS, U. K.

E-mail address: colva.roney-dougal@st-andrews.ac.uk 